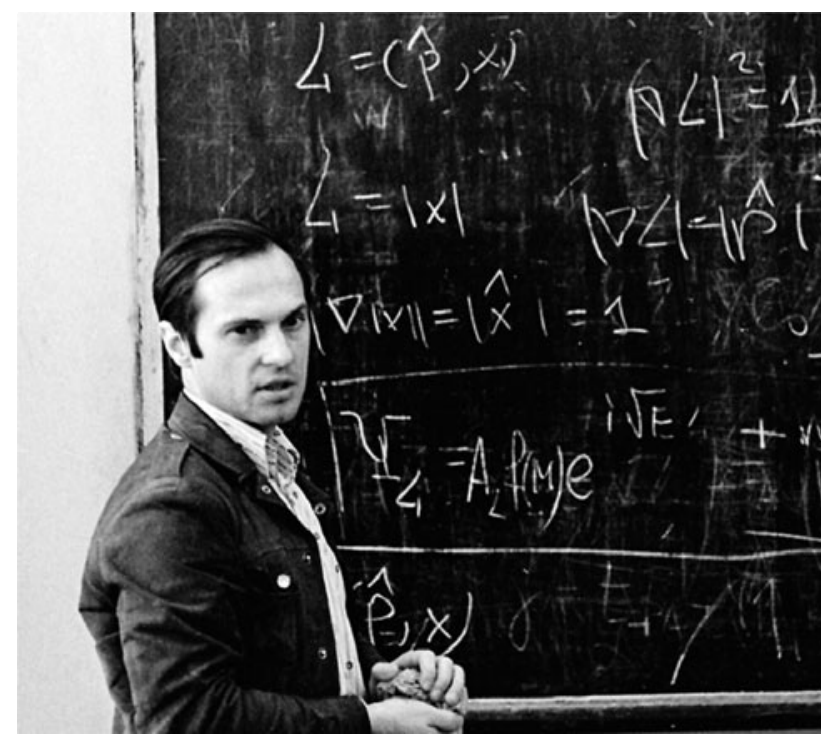

\title{
About S. P. Merkuriev
}

DOI: $10.1134 /$ S0040577916010013

If the life of Academician Stanislav Petrovich Merkuriev had not abruptly ended in 1993 when he was only 48 , he would have been 70 in 2015 . His relatively short life left an imprint on science. His investigations into the quantum theory of several-body systems have become classic. The most prominent results include spectral identities for three-particle scattering matrices and their applications to virial expansions in statistical physics, the study of asymptotic properties of wave functions of three-particle systems in configuration space, and their use for deriving variational principles for three-particle reaction amplitudes, solving a number of mathematical and computational problems in the quantum scattering theory of three-particle systems with Coulomb interaction, creating a new approach for solving the $N$-body problem in quantum mechanics based on matrix differential equations in configuration space, solving the three-body problem with singular potentials determined by boundary conditions, and extensively investigating the physics of systems of a few nucleons, the structure of hadrons, and the physics of atomic and mesomolecular systems.

This issue of Theoretical and Mathematical Physics collects the works of several of Merkuriev's students and colleagues. These works can be viewed as a continuation and extension of a number of methods and ideas whose foundation was laid in his work. The authors of papers in this issue expect that these works will properly contribute to preserving the memory of Stanislav Petrovich Merkuriev.

S. L. Yakovlev

Translated from Teoreticheskaya i Matematicheskaya Fizika, Vol. 186, No. 1, pp. 3-4, January, 2016. 